Faculty of Economics and Social Sciences

Department of Economics

\title{
Priorities in the Location of Multiple Public
} Facilities

Olivier Bochet

Sidartha Gordon

09-02

February 2008

\section{DISCUSSION PAPERS}




\title{
Priorities in the Location of Multiple Public Facilities*
}

\author{
Olivier Bochet ${ }^{\dagger}$ and Sidartha Gordon ${ }^{\ddagger}$
}

June 2008

\begin{abstract}
A collective decision problem is described by a set of agents, a profile of single-peaked preferences over the real line and a number $k$ of public facilities to be located. We consider public facilities that do not suffer from congestion and are non-excludable. We provide a characterization of the class of rules satisfying Pareto-efficiency, object-population monotonicity and sovereignty. Each rule in the class is a priority rule that selects locations according to a predetermined priority ordering among "interest groups". We characterize each of the subclasses of priority rules that respectively satisfy anonymity, hiding-proofness and strategy-proofness. In particular, we prove that a priority rule is strategy-proof if and only if it partitions the set of agents into a fixed hierarchy. Alternatively, any such rule can be viewed as a collection of fixed-populations generalized peakselection median rules (Moulin, 1980), that are linked across populations, in a way that we describe.
\end{abstract}

Keywords: Multiple public facilities, Priority rules, Hierarchical rules, Object-population-monotonicity, Sovereignty, Anonymity, Strategy-proofness, Generalized median rules, Hiding-proofness.

JEL classification codes: D60; D63; D70; D71; H41.

*We thank Anna Bogomolnaia, Haluk Ergin, Lars Ehlers, Bettina Klaus, François Maniquet, Hervé Moulin, Jorge L. Garcia Ramirez, Yves Sprumont and William Thomson for helpful conversations or comments. Part of this paper was written when the first author visited the Université de Montréal, and when the second author visited Maastricht University. Financial support from CIREQ and METEOR is gratefully acknowledged.

$\dagger$ University of Bern.

†Université de Montréal and CIREQ. 


\section{Introduction}

We consider a generalization of the unidimensional voting model studied by Black (1948) and Moulin (1980). A collective decision problem is described by a set of agents, a profile of single-peaked preferences over the real line, and a number $k$ of public facilities to be located -e.g. public libraries. Each public facility is non-excludable and does not exhibit congestion. Thus, it is a pure public good. Each agent has preferences over sets of locations that reflect the fact that she will use only her preferred facility among the $k$ that are provided. We search for a rule that decides on how to locate the facilities, in any possible collective decision problem. We follow the axiomatic method. In addition to Pareto-efficiency, we search for rules that satisfy two normative properties, which we introduce in this paper, object-population monotonicity and sovereignty.

"Public facilities" should be interpreted in a broad sense. They could be actual facilities, such as wireless towers, to be located in space. They could be varieties of software to be provided to a community of users. In this example, the "locations" represent different program varieties. They could also be the official languages for a linguistically diversified society. We will use this third example, to explain the content of our axioms in a concrete manner.

The European Union (henceforth, EU) has to select a set of official working languages for its main tribune, among the set of all the languages used in EU countries. ${ }^{1}$ Not all these languages can be selected as official working language. How should the EU decide which languages to select as official working ones? In addition, how should it modify this selection after an enlargement? We would like to have a method for selecting official working languages that respects a

\footnotetext{
${ }^{1}$ Currently, the official working languages of the EU are English, French and German. For a more detailed discussion concerning the choice of official languages in the EU, see the paper by Fidrmuc, Ginsburgh and Weber (2006).
} 
few desirable properties. ${ }^{2}$ As a first requirement, we require Pareto-efficiency. The selected set of languages should be such that no other set with the same cardinality is weakly preferred by all EU citizens and strictly preferred by at least one citizen. In addition, we are interested in two new properties -objectpopulation monotonicity and sovereignty. Roughly speaking, object-population monotonicity says that if a new group of citizens joins the EU, and at the same time, the number of official working languages increases, in such a way that the increase of the number of languages compensates the increase in linguistic diversity in the EU due to the arrival of new citizens, then all agents initially present should weakly benefit from the change. Last, suppose that a single language needs to be selected as communicating language within EU institutions. Sovereignty loosely says that any language could be selected as the unique working language, provided that an appropriately selected, and possibly large, "group of interest" defending this particular language, is brought into the EU.

Equipped with these axioms, we provide a complete characterization of the class of rules that jointly satisfy them. A priority rule operates as follows. First, it partitions the EU population into linguistic groups, i.e. groups of citizens that share the same preferred language. Second, it ranks these linguistic groups according to some predetermined priority order that can depend on the identities of their members and their full preferences over all languages. The rule then selects the preferred languages of the top $k$ groups in the priority order. Our main result is that any rule that satisfies our three axioms is a priority rule (Theorem 1). All priority rules are efficient, but not all are object-population monotonic nor sovereign. We characterize the class of priority rules that satisfy all three properties (Theorem 1). Priority rules form a rich class. In particular, it includes an interesting subclass of majoritarian rules that rank linguistic

\footnotetext{
${ }^{2}$ In the process of its enlargement, the EU may repeatedly face the choice of adding new languages. Hence, we need a method -a rule in the language of collective choice- that can be used in different situations.
} 
groups according to their cardinality.

Next, we investigate on priority rules that satisfy additional desirable properties, such as anonymity, hiding-proofness, median-selection and strategy-proofness. Unfortunately, no priority rule selects the median voter when a single language needs to be selected and the number of agents is odd. The priority rules that are strategy-proof form an interesting subclass of hierarchical rules (Theorem 2). Each such rule partitions the EU population into a fixed hierarchy of priority levels. Each such rule can also be described as a linked collection of generalized peak-selecting median rules (Moulin, 1980) restricted in a particular way. We provide a complete description of the linkage across populations imposed by our axioms on such a collection.

The problem of locating a single facility is well-studied in the voting literature. Moulin (1980), Ching (1997), Barberà and Jackson (1994) among others, have studied the strategic properties of rules for locating a single facility. Other scholars have studied rules for this problem, that satisfy normative properties. The principle of solidarity says that when circumstances change, all agents not responsible for the change should be affected in the same direction. ${ }^{3}$ Thomson (1993), Ching and Thomson (1996), Vohra (1998) and Klaus (2001) investigate the two main formulations in this context. ${ }^{4}$ In all the papers cited in this paragraph, the rules select the location of a single facility, hence resources are kept fixed.

\footnotetext{
${ }^{3}$ Thomson $(1995,1999 a, 1999 b)$ provides excellent surveys on the different formulations of the solidarity principle and its applications to different economic contexts. Replacementdomination operates on preference profiles for a fixed population. It says that when the preferences of one agent change, all the other agents, whose preferences are kept fixed, should be affected in the same direction. Population-monotonicity operates on preference profiles for a variable population. It says that when new agents join the economy, all the agents who were initially present should all be affected in the same direction.

${ }^{4}$ Gordon (2007b) studies the solidarity principle in the location of a public facility on a cycle. Gordon (2007b) generalizes this literature on the solidarity principle to a more abstract non-geometric setting that contains location problems of single and multiple facilities as special cases.
} 
The problem of locating two facilities was studied first by Miyagawa (1998, 2001). In these works, Miyagawa proposes to extend preferences over single locations to finite sets of locations according to the max-extension. This extension compares finite sets of locations by comparing only their best element. Ehlers (2002, 2003) also studies this problem, but extends preferences in a lexicographic manner. Both Miyagawa $(1998,2001)$ and Ehlers $(2002,2003)$ follow the normative route. They look for rules that satisfy either replacement-domination or population-monotonicity. But once again, the set of resources is kept fixed, since their rules always locate exactly two facilities. In contrast, we do not restrict the set of facilities to any particular cardinality. Thus, our rules prescribe how to locate any number of facilities. In addition, our object-population monotonicity axiom operates across different populations and across problems with different numbers of facilities. It can be viewed as a formulation of the solidarity principle in a context where both population and resources can vary. Our work resembles the work of these scholars in style, in the sense that we also characterize families of rules using axioms, but the axioms we study are different, and the set of location problems we cover is larger.

Barberà and Beviá (2002, 2005) and Ju (2007) also study the location of multiple facilities. They focus on consistency. This property says that when one $k-1$ facility and its "users" are simultaneously removed, the remaining location should remain unchanged. These authors show that the set of rules that satisfy this property and additional interesting requirements is large, but they do not provide a characterization. In contrast, we obtain several characterizations on the basis of object-population monotonicity and other axioms.

Our main contribution is that we identify and axiomatize a subclass of the class of priority rules, in an environement for which few rules have been proposed. These rules have a simple parametric structure. The structure, in turn, facilitates further analysis: It is relatively easy to characterize the set of priority 
rules that satisfy an additional axiom. To obtain our main characterization, we introduce two new axioms. These axioms are meaningful in the context of the location of public facilities, but they also are of independent interest, and could be futher investigated in other contexts.

The plan of the paper is as follows. In Section 2, we introduce the model and necessary definitions. In Section 3, we introduce priorities and priority rules. In Section 4, we present various examples of priorities and priority rules. In Section 5, we present our main axioms. In Section 6, we present our main characterization. In Section 7, we study the subclasses of priority rules that satisfy respectively anonymity, and hiding-proofness. We also show that no priority rule selects the median in problems with one good and a population with an odd cardinality. In Section 8, we study the subclass of strategy-proof priority rules.

\section{The model}

The following definitions are useful throughout the paper. A binary relation over a set $Z$ is a subset of $Z^{2}$. A binary relation $r$ over $Z$ is transitive if for all $x, y, z \in Z$, we have $(x r y$ and $y r z) \Longrightarrow(x r z)$. It is reflexive if for all $x \in Z$, we have $x r x$. It is complete if for all $x, y \in Z$, we have either $x$ r $y$ or $y r x$. It is symmetric if for all $x, y \in Z$, we have $(x r y) \Longrightarrow(y r x)$. It is asymmetric if for all $x, y \in Z$, we have $(x r y) \Longrightarrow \neg(y r x)$. A binary relation over $Z$ is a weak ordering over $Z$ if it is transitive, reflexive and complete. It is a strict ordering over $Z$ if it is transitive, asymmetric and complete. It is a weak partial order if it is transitive and reflexive. It is an indifference relation if it is transitive, reflexive, and symmetric. We will later introduce other types of binary relations.

There is a countably infinite set $\mathbb{N}$ of potential agents. A population $N$ is a finite and nonempty subset of $\mathbb{N}$. The population is collectively endowed with a 
number $k$ of identical public facilities, each to be located on the real line $\mathbb{R}$. A typical location on $\mathbb{R}$ is denoted by $x$. An assignment is a menu of locations, i.e. a finite subset $X \subset \mathbb{R}$. A $k$-assignment is an assignment for exactly $k$ facilities, i.e. a subset $X \subset \mathbb{R}$ such that $|X|=k$. Let $\mathcal{X}_{k}$ be the class of all $k$-assignments. In particular, a 1-assignment is a single location $x \in \mathbb{R}$, so that $\mathcal{X}_{1}=\mathbb{R}$. Let $\mathcal{X} \equiv \cup_{k \geq 1} \mathcal{X}_{k}$ be the class of all assignments.

A preference over $\mathcal{X}$ is a weak ordering over $\mathcal{X}$. Each agent $i \in N$ has a preference $R_{i}$ over $\mathcal{X}$. For each preference $R_{i}$, let $P_{i}$ and $I_{i}$ stand for the strict ordering and the indifference relation associated with $R_{i}$, respectively. We restrict attention to the class $\mathcal{R}$ of single-peaked preferences over $\mathcal{X}$, defined by the following two conditions. The first condition is the common single-peakedness notion, for preferences over single locations on the real line. The second condition extends preferences from single locations to menus. ${ }^{5}$ A preference $R_{i}$ is single-peaked if the following holds.

i) There is a location $p\left(R_{i}\right)$, such that for all $x, y \in \mathbb{R}$ satisfying either $x<y \leq p\left(R_{i}\right)$ or $p\left(R_{i}\right) \geq y>x$, we have $y P_{i} x$. The location $p\left(R_{i}\right)$ is called the peak of preference $R_{i}$.

ii) For all $X, Y \in \mathcal{X}$, we let $X R_{i} Y$ if there is $x \in X$ such that for all $y \in Y$, we have $x R_{i} y$.

For each population $N$, a preference profile for $N$ is a list $R_{N}=\left(R_{i}\right)_{i \in N} \in$ $\mathcal{R}^{N}$. More generally, a preference profile is a preference profile for some population $N .{ }^{6}$ For each profile $R_{N}$ and each subpopulation $K \subseteq N$, let $R_{K}$ denote the subprofile $\left(R_{i}\right)_{i \in K}$. For each profile $R_{N} \in \mathcal{R}^{N}$, let $p\left(R_{N}\right)$ be the set of peak locations for $R_{N}$, i.e. $p\left(R_{N}\right) \equiv\left\{p\left(R_{i}\right): i \in N\right\}$. For each $k>0$, let $\mathcal{P}_{k}$ be the

\footnotetext{
${ }^{5}$ There are different ways to extend preferences over points to preferences over sets. Consistent with the definition of a public facility used in this paper, we consider the max-extension of preferences used by Miyagawa (2001).

${ }^{6}$ A profile $R_{N}$ specifies a population $N$ and each of its agents' preferences.
} 
set of preference profiles $R_{N}$ with a number of distinct peak locations greater than or equal to $k$, i.e. such that $k \leq\left|p\left(R_{N}\right)\right|$. A problem is a pair $\left(k, R_{N}\right)$ such that $k$ is a positive integer, and $R_{N} \in \mathcal{P}_{k} \cdot{ }^{7}$

A rule is a sequence $f=\left\{f_{1}, f_{2}, \ldots\right\}$ of mappings $f_{k}: \mathcal{P}_{k} \rightarrow \mathcal{X}_{k}$. For each problem $\left(k, R_{N}\right)$, the rule $f$ prescribes an assignment in $\mathcal{X}_{k}{ }^{8}$ For each $k \geq 1$, the set of mappings $f_{k}$ is $\mathcal{X}_{k}^{\mathcal{P}_{k}}$. Therefore, the set of all rules is the set $\prod_{k=1}^{\infty} \mathcal{X}_{k}^{\mathcal{P}_{k}}$.

\section{Priority rules}

Let us introduce a class $\mathcal{F}$ of rules which will play an important role in our results. A profile $R_{M}$ is peak-unanimous if all the preferences of this profile have the same peak, i.e. $p\left(R_{M}\right)$ is a singleton. Let $\mathcal{T}$ be the set of peak-unanimous profiles. For any two peak-unanimous profiles $R_{L}$ and $R_{M}^{\prime}$, we say that $R_{L}$ and $R_{M}^{\prime}$ are compatible if they have distinct peaks and disjoint populations, i.e. $p\left(R_{L}\right) \neq p\left(R_{M}^{\prime}\right)$ and $L \cap M=\emptyset$.

We now introduce the class of priorities over some nonempty subset $\mathcal{S}$ of $\mathcal{T}$. Let $\succ$ be a binary relation over $\mathcal{S}$. The binary relation $\succ$ is almost complete if for all $R_{L}, R_{M} \in \mathcal{S}$, we have $\left(R_{L} \succ R_{M}\right.$ or $\left.R_{M} \succ R_{L}\right) \Leftrightarrow\left(R_{L}\right.$ and $R_{M}$ are compatible).$^{9}$ It is almost transitive if for all $R_{K}, R_{L}, R_{M} \in \mathcal{S}$, such that $R_{K}$ and $R_{M}$ are compatible, we have $\left(R_{K} \succ R_{L}\right.$ and $\left.R_{L} \succ R_{M}\right) \quad \Longrightarrow \quad\left(R_{K} \succ R_{M}\right)$. The binary relation $\succ$ is a priority over $\mathcal{S}$ if it is asymmetric, almost transitive and almost complete. ${ }^{10}$ For each nonempty $\mathcal{S} \subseteq \mathcal{T}$, let $\mathbb{P}_{\mathcal{S}}$ be the set of priorities over $\mathcal{S}$.

\footnotetext{
${ }^{7}$ The restriction $k \leq\left|p\left(R_{N}\right)\right|$ allows us to focus on non-trivial cases. When $k>\left|p\left(R_{N}\right)\right|$, it is possible to locate one facility at each peak location, so that the welfare of each agent is maximized. Locating the remaining facilities does not affect any agent's welfare.

${ }^{8}$ Our definitions rule out locating more than one facility at the same point. Under singlepeaked preferences, and for the class of problems we consider, Pareto-efficiency would exclude duplication anyway.

${ }^{9}$ In particular, an almost complete binary relation $\succ$ over $\mathcal{T}$ is never reflexive.

${ }^{10} \mathrm{~A}$ priority $\succ$ is not a partial order, as it is not fully transitive. However, priorities have the following important property. The restriction of a priority $\succ$ on any set $\mathcal{S}$ of pairwise compatible unanimous profiles is a strict ordering. If this set is finite, the priority $\succ$ has a
} 
For each profile $R_{N}$, the peak-unanimous subprofile $R_{M}$ of $R_{N}$ is maximal if $p\left(R_{M}\right) \cap p\left(R_{N \backslash M}\right)=\emptyset$. Any two distinct maximal peak-unanimous subprofiles are compatible. It follows that the collection of maximal peak-unanimous subprofiles of some profile is strictly ordered by any priority (see footnote 10). We are now ready to define the family of priority rules, parametrized by the set $\mathbb{P}_{\mathcal{T}}$. For each $\succ \in \mathbb{P}_{\mathcal{T}}$, the priority rule $f$ associated with $\succ$ is defined as follows. Let $\left(k, R_{N}\right)$ be an arbitrary problem. Then the priority $\succ$ strictly ranks the maximal peak-unanimous subprofiles in the decomposition of $R_{N}$ and $f_{k}\left(R_{N}\right)$ selects the peak locations of the top $k$ maximal peak-unanimous subprofiles for $\succ$. In more precise terms, $f_{k}\left(R_{N}\right)$ is the $k$-assignment such that $f_{k}\left(R_{N}\right) \subseteq p\left(R_{N}\right)$, and for all two maximal peak-unanimous subprofiles $R_{M}$ and $R_{L}$ in $R_{N}$, if $p\left(R_{M}\right) \subseteq f_{k}\left(R_{N}\right)$ and $p\left(R_{L}\right) \varsubsetneqq f_{k}\left(R_{N}\right)$, then $R_{M} \succ R_{L}$. Let $\mathcal{F}$ be the set of priority rules.

\section{Examples}

In this section, we provide a few examples that illustrate how large and diverse the set of priorities is. We first present some priorities that can be described in a simple way (Examples 1, 2 and 3), and then move on to more complex priorities, for which it is convenient to use a lexicographic formulation (Examples 4 to 8).

Example 1: The left-peaks priority $\succ^{L P}$ is such that, for all compatible $R_{M}$, $R_{N} \in \mathcal{T}$, we have $R_{M} \succ^{L P} R_{N}$ if and only if $p\left(R_{M}\right)<p\left(R_{N}\right)$. The left-peaks rule $f^{L P}$ is the priority rule defined by $\succ^{L P}$. An alternative definition of the rule $f^{L P}$ is that it selects, for each problem $\left(k, R_{N}\right)$, the $k$-assignment $X$ such that for all $i \in N$, if $p\left(R_{i}\right) \notin X$, then $p\left(R_{i}\right)>\max (X)$.

greatest (or top) element in $\mathcal{S}$. A top element for $\succ$ typically does not generally exist on a set of unanimous profiles whose elements are not pairwise compatible, even if it is a finite set. 
Example 2: The right-peaks priority $\succ^{R P}$ is such that, for all compatible $R_{M}$, $R_{N} \in \mathcal{T}$, we have $R_{M} \succ^{R P} R_{N}$ if and only if $p\left(R_{M}\right)>p\left(R_{N}\right)$. The right-peaks rule $f^{R P}$ is the priority rule defined by $\succ^{R P}$. An alternative definition of the rule $f^{R P}$ is that it selects, for each problem $\left(k, R_{N}\right)$, the $k$-assignment $X$ such that for all $i \in N$, if $p\left(R_{i}\right) \notin X$, then $p\left(R_{i}\right)<\min (X){ }^{11}$

Example 3: A priority $\succ^{S D}$ is a serial dictatorship if there exists a strict ordering $\triangleright$ of all agents in $\mathbb{N}$ such that, for all compatible $R_{M}, R_{N} \in \mathcal{T}$, we have $R_{M} \succ^{S D} R_{N}$ if and only if there exists $i \in M$, such that for all $j \in N$, we have $i \triangleright j$. For example, the strict ordering could be such that for all $i, j \in \mathbb{N}$, we have $i \triangleright j$ if and only if $i<j .^{12}$

Lexicographic formulations: Some priorities are easier to describe using a lexicographic formulation. This means that the priority is determined first by a primary criterion that is not always decisive. When the primary criterion is indecisive, a secondary criterion is used, which can vary depending on the pair that is being compared.

To formalize this idea, let us introduce the following definitions. Let $\succeq$ be a weak ordering over $\mathcal{T}$. Let $\mathbb{T}$ be the set of indifference classes for $\succeq$ in $\mathcal{T}{ }^{13}$ For each $T \in \mathbb{T}$, let $\succ_{T}$ be a priority over $T$. Finally, let $\succ$ be a priority over $\mathcal{T}$. Then, the priority $\succ$ has the lexicographic formulation $\left(\succeq,\left(\succ_{T}\right)_{T \in \mathbb{T}}\right)$ if for all compatible $R_{M}, R_{N} \in \mathcal{T}$, we have $R_{M} \succ R_{N}$ if and only if $R_{M} \succeq R_{N}$ and

\footnotetext{
${ }^{11}$ Miyagawa (2001) showed that when $|N| \geq 4$ and $k=2$, the only mappings $\mathcal{R}^{N} \rightarrow \mathcal{X}_{2}$ satisfying efficiency and replacement-domination are the left-peaks rule and the right-peaks rule. These solutions are also anonymous and group-strategy-proof. The left-peaks and rightpeaks priorities are also trivial hierarchical rules (see Example 4) where all agents belong to the same unique indifference class.

${ }^{12}$ The serial dictatorships are extreme cases of hierarchical rules (see Example 4) where each indifference class is a singleton.

${ }^{13}$ Requiring $\succeq$ to be a weak ordering rather than an almost complete and almost transitive (and not asymetric) binary relation on $\mathcal{T}$ is important here, as it allows us to refer to the indifference classes of $\succeq$.
} 
either (i) $\neg\left(R_{N} \succeq R_{M}\right)$ or (ii) There is an indifference class $T$ for $\succeq$ such that $\left(R_{M}, R_{N} \in T\right.$ and $\left.R_{M} \succ_{T} R_{N}\right)$.

It should be clear that all priorities admit many lexicographic formulations. However, there are priorities for which a particular lexicographic formulation is the most natural and convenient definition. We now present a few examples of such priorities.

Example 4: A priority $\succ^{H}$ is hierarchical if it admits a lexicographic formulation $\left(\succeq,\left(\succ_{T}\right)\right)$ that satisfies the following conditions. (i) There is a weak ordering $\unrhd$ of all agents in $\mathbb{N}$, such that, for all $R_{M}, R_{N} \in \mathcal{T}$, we have $R_{M} \succeq R_{N}$ if and only if there exists $i \in M$, such that for all $j \in N$, we have $i \unrhd j$. (ii) For each $T \in \mathbb{T}$, the priority $\succ_{T}$ is either the (restriction to $T$ of) the left-peaks or (restriction to $T$ of) the right-peaks priority. ${ }^{14}$

Example 5: A priority $\succ^{M}$ is majoritarian if it admits a lexicographic formulation $\left(\succeq,\left(\succ_{n}\right)\right)$ such that, for all $R_{M}, R_{N} \in \mathcal{T}$, we have $R_{M} \succeq R_{N}$ if and only if $|M| \geq|N|$. The indifference classes are the sets $T_{n}=\left\{R_{N} \in \mathcal{T}:|N|=n\right\}$. For each $n \geq 1$, the tie-breaking rule $\succ_{n}$ can be any priority. For example, we could require $\succ_{n}$ to be the left-peaks priority, for all $T$ (left-majoritarian priority) or the right-peaks priority (right-majoritarian priority). ${ }^{15}$

Example 6: A priority $\succ^{M}$ is $x_{0}$-centralist-majoritarian if it admits a lexicographic formulation $\left(\succeq,\left(\succ_{v}\right)\right)$, such that there are a location $x_{0} \in \mathbb{R}$ (the "center"), a distance $D(x, y)$ on $\mathbb{R}$ and an index $u:\{1,2, \ldots\} \times \mathbb{R}_{+} \rightarrow \mathbb{R}$, where $u(n, d)$ is increasing in $n$ and decreasing in $d$, such that, for all $R_{M}, R_{N} \in \mathcal{T}$,

\footnotetext{
${ }^{14}$ In Section 9, we show that hierarchical rules are the only priority rules that are strategyproof. One could also define a more general class of (non strategy-proof) hierarchical rules by allowing the $\succ_{T}$ to be any priority, non necessarily the left-peaks or right-peaks priorities.

${ }^{15}$ We can similarly define the family of minoritarian priorities by requiring instead that $R_{M} \succeq R_{N}$ iff $|M| \leq|N|$.
} 
we have $R_{M} \succeq R_{N}$ if and only if

$$
u\left(|M|, D\left(p\left(R_{M}\right), x_{0}\right)\right) \geq u\left(|N|, D\left(p\left(R_{N}\right), x_{0}\right)\right) .
$$

The indifference classes are the sets

$$
T_{v}=\left\{R_{N} \in \mathcal{T}: u\left(|M|, D\left(p\left(R_{M}\right), x_{0}\right)\right)=v\right\} .
$$

For each such class, the tie-breaking rule $\succ_{v}$ can be any priority. For example, we could require $\succ_{v}$ to be the left-peaks priority for all $v \in \mathbb{R}$ (left- $x_{0^{-}}$ centralist-majoritarian priority) or the right-peaks priority for all $v \in \mathbb{R}$ (right$x_{0}$-centralist-majoritarian priority). ${ }^{16}$

We conclude this section by an illustration of how the left-majoritarian rule $f^{L M}$ allocates goods for two particular problems. Let $N=\{1, \ldots, 10\}, k=2$ and the two profiles $R_{N}$ and $R_{N}^{\prime}$ with peak locations distributed as follows.

\begin{tabular}{|c|c|c|c|c|}
\hline Profile $R_{N}$ & Agents & 9,10 & $6,7,8$ & $1, \ldots, 5$ \\
\hline & Peak locations & 0 & 1 & 2 \\
\hline
\end{tabular}

\begin{tabular}{|c|c|c|c|c|c|}
\hline Profile $R_{N}^{\prime}$ & Agents & $1,2,3$ & $4,5,6$ & $7,8,9$ & 10 \\
\hline & Peak locations & 0 & 1 & 2 & 3 \\
\hline
\end{tabular}

Let $R_{L_{0}}, R_{L_{1}}$ and $R_{L_{2}}$ be the maximal peak-unanimous subprofiles of $R_{N}$, with $L_{0}=\{9,10\}, L_{1}=\{6,7,8\}$, and $L_{2}=\{1, \ldots, 5\}$, we have $\left|L_{0}\right|<\left|L_{1}\right|<$ $\left|L_{2}\right|$, therefore $R_{L_{2}} \succ^{L M} R_{L_{1}} \succ^{L M} R_{L_{0}}$, i.e. $f_{2}\left(R_{N}\right)=\{1,2\}$. Similarly, let $R_{M_{0}}^{\prime}, R_{M_{1}}^{\prime} R_{M_{2}}^{\prime}$ and $R_{M_{2}}^{\prime}$ be the maximal peak-unanimous subprofiles of $R_{N}^{\prime}$, with $M_{0}=\{1,2,3\}, M_{1}=\{4,5,6\}, M_{2}=\{7,8,9\}$, and $M_{3}=\{10\}$, we have $\left|M_{0}\right|=\left|M_{1}\right|=\left|M_{2}\right|>\left|M_{3}\right|$, and $p\left(R_{M_{0}}^{\prime}\right)<p\left(R_{M_{1}}^{\prime}\right)<p\left(R_{M_{2}}^{\prime}\right)$, thus $R_{M_{0}}^{\prime} \succ^{L M} R_{M_{1}}^{\prime} \succ^{L M} R_{M_{2}}^{\prime} \succ^{L M} R_{M_{3}}^{\prime}$, i.e. $f_{2}^{L M}\left(R_{N}^{\prime}\right)=\{0,1\}$.

\footnotetext{
${ }^{16} \mathrm{~A}$ more general family is obtained by relaxing the monotonicity asumptions on $u$.
} 


\section{Axioms}

Our first axiom is the usual (Pareto)-efficiency axiom. For each profile $R_{N} \in$ $\mathcal{R}^{N}$ and each $x, y \in \mathbb{R}$, we say that $x$ weakly Pareto-dominates $y$ for profile $R_{N}$ if $x R_{i} y$ for each $i \in N$. This is denoted by $x R_{N} y$.

A rule $f$ satisfies efficiency if, for each problem $\left(k, R_{N}\right)$, there is no $k$ assignment $X$ such that $X R_{N} f_{k}\left(R_{N}\right)$, and $X P_{j} f_{k}\left(R_{N}\right)$ for some $j \in N$.

Any rule that always selects distinct peaks locations is efficient. Therefore, all priority rules are efficient. Next, we would like our rules to satisfy a fairness condition when resources and population vary. First, when the population is held fixed but resources vary, it is natural to require that the agents who are initially present in the economy, and whose preferences are kept fixed, all weakly gain as the number of available facilities increases.

A rule satisfies object-monotonicity if, for each problem $\left(k, R_{N}\right)$ such that $k<\left|p\left(R_{N}\right)\right|$, we have $f_{k+1}\left(R_{N}\right) R_{N} f_{k}\left(R_{N}\right)$.

All priority rules are object-monotonic, since they satisfy, for each such problem, the stronger condition $f_{k}\left(R_{N}\right) \subset f_{k+1}\left(R_{N}\right)$. Object-monotonicity turns out to be very weak in this model. Many rules that have very little in common satisfy this axiom. This motivates looking at changes in both resources and population. ${ }^{17}$ We require that if both resources and population increase, and the new resources are sufficient to fully satisfy the newcomers, then the agents who are initially present in the economy, and whose preferences are kept fixed,

\footnotetext{
${ }^{17}$ One could also study changes in population, while resources are kept fixed. The natural axiom for this case, population monotonicity, requires that when new agents join the economy (for a fixed number of facilities), all the agents that were initially present weakly lose. This property is studied in this model by Miyagawa (1998), and by Ehlers (2003) in a model similar to this one. Both restrict attention to the case of two facilities. Gordon (2007a) shows that this axiom has strong general implications in the broader class of pure public goods, of which this model is a special case.
} 
all weakly gain from this double expansion. In fact, we will restrict attention to changes where exactly one facility is brought into the economy and exactly one peak-unanimous profile is added to the initial profile. Observe that the additional facility is indeed sufficient to fully satisfy the newcomers, since it could be located at their common peak.

A rule $f$ satisfies object-population monotonicity if, for each problem $\left(k, R_{N}\right)$ with $k<\left|p\left(R_{N}\right)\right|$, for each peak-unanimous profile $R_{M} \in \mathcal{T}$ such that $N \cap M=\emptyset$, we have $f_{k+1}\left(R_{N}, R_{M}\right) R_{N} f_{k}\left(R_{N}\right)$.

All the priority rules presented in Examples 1 to 6 satisfy this axiom. This is because the priority relations that define them have the following property. A priority $\succ$ is monotonic if, for all peak-unanimous profiles $R_{L}, R_{N}$, and any agent $i \notin N \cup L$, we have $R_{N} \succ R_{L} \Rightarrow R_{N \cup\{i\}} \succ R_{L}$. In fact, any priority rule whose priority is monotone satisfies object-population monotonicity. Priority rules whose priority is not monotone may not satisfy object-population monotonicity, as shown in the following example.

Example 7: Let $\succ$ be the priority such that for all two compatible peakunanimous profiles $R_{L}$ and $R_{M}$, we have $R_{L} \succ R_{M}$ if either $(|L|>|M|$ and $|L| \geq 3)$ or $(|L|=1$ and $|M|=2)$ or $\left(|L|=|M|\right.$ and $\left.p\left(R_{L}\right)<p\left(R_{M}\right)\right)$. The priority rule $f$ defined by $\succ$ violates object-population monotonicity. To see this, consider a profile $R_{\{1,2,3,4\}}$ such that $p\left(R_{1}\right)=p\left(R_{2}\right)<p\left(R_{3}\right)<p\left(R_{4}\right)$. Therefore $R_{1} \succ R_{3} \succ R_{4} \succ R_{\{1,2\}}$. Thus, $f_{1}\left(R_{\{1,3,4\}}\right)=p\left(R_{1}\right)$ and $f_{2}\left(R_{\{1,2,3,4\}}\right)=$ $\left\{p\left(R_{3}\right), p\left(R_{4}\right)\right\}$. Therefore, $f_{1}\left(R_{\{1,3,4\}}\right) P_{1} f_{2}\left(R_{\{1,2,3,4\}}\right)$, in contradiction with object-population monotonicity.

The exact necessary and sufficient condition for a priority to define a rule that is object-population monotonic is the following. A priority $\succ$ is almost monotonic if, there are no four peak-unanimous profiles $R_{M}, R_{K}, R_{H}$ and $R_{L}$ 
such that $p\left(R_{M}\right)=p\left(R_{L}\right), M \cap L=\emptyset, R_{K}$ and $R_{H}$ are compatible, $R_{M} \succ$ $R_{H} \succ R_{M \cup L}$, and $R_{M} \succ R_{K} \succ R_{M \cup L}$. In the proof of Theorem 1 (step 4), we will verify that this condition on the priority is indeed necessary for a priority rule to be object-population monotonic. We leave it to the reader to check that it is also sufficient.

Example 7 violates object-population monotonicity because the axiom requires that agent 1 , who has the same peak as the newcomer agent 2 , also weakly gain from the change. One can formulate a weaker, but somewhat unnatural, version of the axiom that requires all agents initially present to weakly gain from the addition of exactly one facility and one peak-unanimous profile, except for agents who have the same peak as the newcomers. ${ }^{18}$ All priority rules satisfy this weaker version of the axiom (Bochet and Gordon, 2008). We will however proceed with the stronger version of the axiom, which we feel is more natural and compelling. ${ }^{19}$

A related question is the following. Are all rules that satisfy efficiency and object-population monotonicity priority rules? Again, the answer is negative, as shown in the following example.

Example 8: Let $\triangleright$ be the strict ordering on $\mathbb{R}$ such that, for all $x, x^{\prime}, y, y^{\prime}, z, z^{\prime} \in$ $\mathbb{R}$ satisfying $x<x^{\prime}<0<y<y^{\prime}<1<z<z^{\prime}$, we have $0 \triangleright 1 \triangleright y \triangleright y^{\prime} \triangleright x \triangleright x^{\prime} \triangleright z \triangleright$

\footnotetext{
${ }^{18} \mathrm{~A}$ precise definition of this weaker version of the axiom is as follows. A rule $f$ satisfies object-population monotonicity - if, for each problem $\left(k, R_{N}\right)$ with $k<\left|p\left(R_{N}\right)\right|$, for each peak-unanimous profile $R_{M} \in \mathcal{T}$ such that $N \cap M=\emptyset$, and for all $i \in N$ such that $p\left(R_{i}\right) \neq p\left(R_{M}\right)$, we have $f_{k+1}\left(R_{N}, R_{M}\right) R_{N} f_{k}\left(R_{N}\right)$.

${ }^{19}$ Object-population monotonicity does not imply object-monotonicity. However, objectpopulation monotonicity, efficiency and sovereignty do imply object-monotonicity. Objectpopulation monotonicity is also logically independent from population monotonicity. The rules presented in Example 5 satisfy object-population monotonicity, but not populationmonotonicity. Moreover consider the rule $f$ for which for each problem $\left(1, R_{N}\right)$, we have $f_{1}\left(R_{N}\right)=f_{1}^{L P}\left(R_{N}\right)$, and for each $k \geq 2$ and each problem $\left(k, R_{N}\right)$, we have $f_{k}\left(R_{N}\right)=f_{k}^{R P}\left(R_{N}\right)$. This rule satisfies population monotonicity but not object-population monotonicity.
} 
$z^{\prime}$. Let $\succ$ be the priority such that, for any two peak-unanimous profiles $R_{L}$ and $R_{M}^{\prime}$, we have $R_{L} \succ R_{M}^{\prime}$ if and only if $p\left(R_{L}\right) \triangleright p\left(R_{M}^{\prime}\right)$. Let $g$ be the priority rule defined by $\succ$. Let $f$ be the rule such that, for each problem $\left(k, R_{N}\right)$, if $k=1$ and $p\left(R_{N}\right)=\{-1,1\}$, then $f_{k}\left(R_{N}\right) \equiv\left\{\frac{1}{2}\right\}$, and otherwise $f_{k}\left(R_{N}\right) \equiv g_{k}\left(R_{N}\right)$. The rule $f$ satisfies efficiency and object-population monotonicity, but it is not a priority rule.

We wish to eliminate rules that present pathologies of the kind we see in Example 8. This leads us to introduce a third axiom. This axiom only restricts the one-facility component $f_{1}$ of a rule, as the population varies. It says that the choice $y$ made by the rule $f_{1}$ for a given profile $R_{N}$ can be reversed in favor of any given location $x$ provided that some appropriately selected peak-unanimous profile $R_{M}$ is brought into the economy in support of $x$.

A rule $f$ satisfies sovereignty - if, for each profile $R_{N}$, each location $x \in$ $\mathbb{R} \backslash f_{1}\left(R_{N}\right)$, there exists a peak-unanimous profile $R_{M} \in \mathcal{T}$ such that $M \cap N=\emptyset$, that satisfies $f_{1}\left(R_{N}, R_{M}\right)=\{x\}=p\left(R_{M}\right)$.

In fact, we will use the following slightly stronger version of the axiom, which requires that there are infinitely many distinct and compatible such peakunanimous profiles.

A rule $f$ satisfies sovereignty if, for each profile $R_{N}$, each location $x \in \mathbb{R} \backslash$ $f_{1}\left(R_{N}\right)$, and each population $L$, there exists a peak-unanimous profile $R_{M} \in \mathcal{T}$ such that $M$ is disjoint from both $L$ and $N$, that satisfies $f_{1}\left(R_{N}, R_{M}\right)=\{x\}=$ $p\left(R_{M}\right)$.

The rule presented in Example 8 violates both versions of the axiom. We will show in the next section that any efficient, object-population monotonic and sovereign rule is a priority rule. However, not all priority rules are sovereign. While the rules in examples 5, 6 and 7, and some of the rules in examples 3 and 
4 are, the ones in examples 1 and 2, and some of the rules in example 3 and 4 are not. We now present a necessary and sufficient condition on the priority for the priority rule it defines to be sovereign. We say that the priority $\succ$ is sovereign if the following two conditions hold. (i) For all peak-unanimous $R_{H}, R_{K} \in \mathcal{T}$ such that $R_{H} \succ R_{K}$, and for any population $L$, there exists a peak-unanimous profile $R_{M} \in \mathcal{T}$ such that $M$ is disjoint from $K$ and $L$, and satisfies $p\left(R_{M}\right)=p\left(R_{K}\right)$, and $R_{K \cup M} \succ R_{H}$. (ii) For each $R_{H} \in \mathcal{T}$, each $x \neq p\left(R_{H}\right)$ and each population $L$, there exists a peak-unanimous profile $R_{M} \in \mathcal{T}$ such that $M \cap L=\emptyset$, and satisfies $p\left(R_{M}\right)=x$, and $R_{M} \succ R_{H}$. In the proof of Theorem 1 (step 5), we will verify that this condition on the priority is indeed necessary for a priority rule to be sovereign. We leave it to the reader to check that it is also sufficient.

\section{Main characterization}

We are now ready to present our main result.

Theorem 1: A rule $f$ satisfies efficiency, object-population monotonicity and sovereignty if and only if it is a priority rule whose priority is almost monotonic and sovereign. ${ }^{20}$

As we observed in Section 5, it is quite easy to verify that all priority rules whose priority is almost monotone and sovereign satisfy the three axioms. Before proving the converse, we first present two useful lemmas. The first one states that object-population monotonicity and sovereignty imply the following property.

\footnotetext{
${ }^{20}$ In an earlier version of this work (Bochet and Gordon, 2008), we prove the following related result. A rule satisfies efficiency, object-population monotonicity_ (see footnote 18) and sovereignty if and only if it is a priority rule whose priority is sovereign (Theorem 1). Furthermore, the following claims are equivalent for any rule $f$ with domain $\mathcal{D}$ : $(i)$ The rule $f$ is such that for all finite $D \subset \mathcal{D}$, there exists a rule $g_{D}$ on $\mathcal{D}$ that satisfies efficiency, objectpopulation monotonicity - and sovereignty and coincides with $f$ on $D$; $(i i)$ The rule $f$ is a priority rule (Theorem 2).
} 
A rule $f$ satisfies strong sovereignty if for each problem $\left(k, R_{N}\right)$, each location $x \in \mathbb{R} \backslash f_{k}\left(R_{N}\right)$, and each population $L$, there exists a peak-unanimous profile $R_{M}$ such that $M$ is disjoint from both $L$ and $N$, that satisfies $p\left(R_{M}\right)=$ $\{x\} \subseteq f_{k}\left(R_{N}, R_{M}\right)$.

Lemma 1: If $f$ satisfies object-population monotonicity and sovereignty, then it satisfies strong sovereignty

Proof. Let $\left(k, R_{N}\right)$ be an arbitrary problem, let $x \in \mathbb{R}$ be an arbitrary location, and let $L$ be an arbitrary population. We will prove that there exists a peak-unanimous profile $R_{M}$ such that $M \cap(L \cup N)=\emptyset$ and $p\left(R_{M}\right)=\{x\} \subseteq$ $f_{k}\left(R_{N}, R_{M}\right)$. If $k=1$, then sovereignty guarantees that this is true. So suppose instead, that $k>1$. Let $\ell \equiv\left|p\left(R_{N}\right)\right|$. We have $\ell \geq k$.

Let $R_{N_{1}}, \ldots, R_{N_{\ell-(k-1)}}$ be $\ell-(k-1)$ distinct maximal peak-unanimous subprofiles of $R_{N}$. Let $\widetilde{N} \equiv N_{1} \cup \ldots \cup N_{\ell-(k-1)}$. We have $\left|p\left(R_{\widetilde{N}}\right)\right|=\ell-(k-1)$. Let $\widetilde{L} \equiv(N \backslash \widetilde{N}) \cup L$. By sovereignty, there exists a profile $R_{M} \in \mathcal{R}^{M}$ such that $M \cap(\widetilde{L} \cup \widetilde{N})=\emptyset$, and $p\left(R_{M}\right)=\{x\}=f_{1}\left(R_{\widetilde{N}}, R_{M}\right)$. We have $\left|p\left(R_{N \backslash \tilde{N}}\right)\right|=k-1$. By object-population monotonicity, applied $k-1$ times, we have in particular $f_{k}\left(R_{\widetilde{N}}, R_{M}, R_{N \backslash \widetilde{N}}\right) R_{M} f_{1}\left(R_{\widetilde{N}}, R_{M}\right)$. Since $f_{1}\left(R_{N}, R_{M}\right)=\{x\}=p\left(R_{M}\right)$, this implies that $x \in f_{k}\left(R_{\widetilde{N}}, R_{M}, R_{N \backslash \tilde{N}}\right)$, i.e. $p\left(R_{M}\right)=\{x\} \subseteq f_{k}\left(R_{N}, R_{M}\right)$. Since $M \cap(L \cup N)=M \cap(\widetilde{L} \cup \widetilde{N})=\emptyset$, therefore the population $M$ satisfies all the desired properties.

The second lemma shows that the three axioms Theorem 1 imply that each public facility must be located at some agent's peak location.

A rule $f$ satisfies peak-selection if for each problem $\left(k, R_{N}\right)$, we have $f_{k}\left(R_{N}\right) \subseteq p\left(R_{N}\right)$.

Lemma 2: If $f$ satisfies efficiency, object-population monotonicity and sovereignty, then it satisfies peak-selection. 
Proof: Let $f$ satisfy the three axioms. We prove by induction on $k$, that each $f_{k}$ satisfies peak-selection.

Step 1. The rule $f_{1}$ satisfies peaks-selection.

Suppose by contradiction that $f_{1}$ does not satisfy peak-selection. Let $R_{N} \in$ $\mathcal{R}^{N}$ and let $x \in \mathbb{R}$ be such that $f_{1}\left(R_{N}\right)=\{x\}$ and $x \notin p\left(R_{N}\right)$. Throughout the proof, for all $j \in N$, let $p_{j}:=p\left(R_{j}\right)$. By efficiency, there are two agents $i, \ell \in N$ such that $p_{i}<x<p_{\ell}$, and $p\left(R_{N}\right)$ has no element strictly comprised between $p_{i}$ and $p_{\ell}$. Let $u, v \in \mathbb{R}$ be such that $p_{i}<u<x<v<p_{\ell}$. By strong sovereignty, there are peak-unanimous profiles $R_{J}$ and $R_{K}$ satisfying the following conditions. Let $R_{J}$ be such that $J \cap N=\emptyset, p\left(R_{J}\right)=\{u\}$, and $u \in f_{2}\left(R_{N}, R_{J}\right)$. Similarly, let $R_{K}$ be such that $K \cap(N \cup J)=\emptyset, p\left(R_{K}\right)=\{v\}$, and $v \in f_{2}\left(R_{N}, R_{K}\right)$. Let $A \equiv f_{3}\left(R_{N}, R_{J}, R_{K}\right)$. We will now show that $A$ has at least four elements. First, by object-population monotonicity, $p\left(R_{J}\right)=\{u\}$ and $u \in f_{2}\left(R_{N}, R_{J}\right)$ imply that $u \in A$. Similarly, by object-population monotonicity, $p\left(R_{K}\right)=\{v\}$ and $v \in f_{2}\left(R_{N}, R_{K}\right)$ imply that $v \in A$.

Second, by object-population monotonicity, since $f_{1}\left(R_{N}\right)=\{x\}$, then the set $f_{2}\left(R_{N}, R_{K}\right)$ has at least one location that is at least as good as $x$ for $R_{i}$. Let $y \in f_{2}\left(R_{N}, R_{K}\right)$ such that $y R_{i} x$. Efficiency requires that $y \leq p_{i}$. In particular $y<v$. Since $\left|f_{2}\left(R_{N}, R_{K}\right)\right|=2$, it then follows that $f_{2}\left(R_{N}, R_{K}\right)=$ $\{y, v\}$. By efficiency, there exists an agent $h \in N$ such that $p_{h} \leq y$. By objectpopulation monotonicity, since $f_{2}\left(R_{N}, R_{K}\right)=\{y, v\}$, then the set $A$ has at least one location that is at least as good as $y$ for $R_{h}$. Let $y^{\prime} \in A$ such that $y^{\prime} R_{h} y$. Since $p_{h} \leq y$, then in particular $y^{\prime} \leq y<v$.

Third, by object-population monotonicity, since $f_{1}\left(R_{N}\right)=\{x\}$, then the set $f_{2}\left(R_{N}, R_{J}\right)$ has at least one location that is at least as good as $x$ for $R_{\ell}$. Let $z \in f_{2}\left(R_{N}, R_{J}\right)$ such that $z R_{\ell} x$. Efficiency requires that $p_{\ell} \leq z$. In particular $u<z$. Since $\left|f_{2}\left(R_{N}, R_{J}\right)\right|=2$, it then follows that $f_{2}\left(R_{N}, R_{J}\right)=$ $\{u, z\}$. By efficiency, there exists an agent $m \in N$ such that $z \leq p_{m}$. By object- 
population monotonicity, since $f_{2}\left(R_{N}, R_{J}\right)=\{u, z\}$, then the set $A$ has at least one location that is at least as good as $x$ for $R_{m}$. Let $z^{\prime} \in A$ such that $z^{\prime} R_{m} z$. Since $z \leq p_{m}$, then in particular $u<z \leq z^{\prime}$.

In conclusion, $y^{\prime}<u<v<z^{\prime}$ are four distinct elements of $A$, in contradiction with $|A|=3$.

Step 2. Let $k \geq 1$. Suppose that $f_{k}$ satisfies peak-selection. Then $f_{k+1}$ also satisfies peak-selection.

Let $R_{N} \in \mathcal{R}^{N}$. If $\left|p\left(R_{N}\right)\right|=k+1$, then by efficiency, $f_{k+1}\left(R_{N}\right)=p\left(R_{N}\right)$, so the claim is true. Suppose then that $\left|p\left(R_{N}\right)\right|>k+1$. We will first show that $f_{k+1}\left(R_{N}\right) \cap p\left(R_{N}\right) \neq \emptyset$. Since $\left|p\left(R_{N}\right)\right|>k+1$, then $p\left(R_{N}\right) \nsubseteq f_{k+1}\left(R_{N}\right)$. Let $R_{M}$ be a maximal peak-unanimous subprofile of $R_{N}$, with $M \subset N$, such that $p\left(R_{M}\right) \notin f_{k+1}\left(R_{N}\right)$. Consider the profile $R_{N \backslash M}$. We have $\left|p\left(R_{N \backslash M}\right)\right|>k$. From the induction hypothesis, we have $f_{k}\left(R_{N \backslash M}\right) \subseteq p\left(R_{N \backslash M}\right)$. By objectpopulation monotonicity, we have $f_{k}\left(R_{N \backslash M}\right) \subseteq f_{k+1}\left(R_{N}\right)$. Thus $f_{k}\left(R_{N \backslash M}\right) \subseteq$ $f_{k+1}\left(R_{N}\right) \cap p\left(R_{N}\right)$. Therefore, $f_{k+1}\left(R_{N}\right) \cap p\left(R_{N}\right) \neq \emptyset$.

Let $x \in f_{k+1}\left(R_{N}\right) \cap p\left(R_{N}\right)$. Let $R_{L}$ be the maximal peak-unanimous subprofile of $R_{N}$ such that $p\left(R_{L}\right)=\{x\}$. Then $\left|p\left(R_{N \backslash L}\right)\right|>k$ and $x \notin p\left(R_{N \backslash L}\right)$. From the induction hypothesis, we have $f_{k}\left(R_{N \backslash L}\right) \subseteq p\left(R_{N \backslash L}\right)$. By objectpopulation monotonicity, we have $f_{k}\left(R_{N \backslash L}\right) \subseteq f_{k+1}\left(R_{N}\right)$. Since $x \in f_{k+1}\left(R_{N}\right)$, then $f_{k}\left(R_{N \backslash L}\right) \cup\{x\} \subseteq f_{k+1}\left(R_{N}\right)$. Since $x \notin p\left(R_{N \backslash L}\right)$, then $x \notin f_{k}\left(R_{N \backslash L}\right)$. Thus, $\left|f_{k}\left(R_{N \backslash L}\right) \cup\{x\}\right|=k+1=\left|f_{k+1}\left(R_{N}\right)\right|$. Therefore, $f_{k+1}\left(R_{N}\right)=f_{k}\left(R_{N \backslash L}\right) \cup$ $\{x\}$. Therefore, $f_{k+1}\left(R_{N}\right) \subseteq p\left(R_{N}\right)$, the desired conclusion.

We are now ready to prove Theorem 1.

Proof of Theorem 1 (only if implication): Let $f$ be an arbitrary rule that satisfies efficiency, object-population monotonicity and sovereignty.

Step 1: Construction of a candidate priority $\succ$ from $f$. 
By peak-selection, for each two compatible peak-unanimous profiles $R_{L}$ and $R_{M}$, we have $f_{1}\left(R_{L}, R_{M}\right) \subset p\left(R_{L}\right) \cup p\left(R_{M}\right)$. Let $\succ$ be the binary relation over peak-unanimous profiles such that, for each two compatible peak-unanimous profiles $R_{L}$ and $R_{M}$, we have $R_{L} \succ R_{M}$ if $f_{1}\left(R_{L}, R_{M}\right)=p\left(R_{L}\right)$. By construction, the relation $\succ$ is asymmetric and almost complete. It remains to show that $\succ$ is almost transitive. Consider three arbitrary peak-unanimous profiles $R_{K}, R_{L}$ and $R_{M}$ such that $R_{K}$ and $R_{M}$ are compatible. Suppose that $R_{K} \succ R_{L}$ and $R_{L} \succ R_{M}$. Then in particular, $R_{K}$ and $R_{L}$ are compatible, and $R_{L}$ and $R_{M}$ are compatible. By definition of $\succ$, we know that $f_{1}\left(R_{K}, R_{L}\right)=p\left(R_{K}\right)$ and $f_{1}\left(R_{L}, R_{M}\right)=p\left(R_{L}\right)$. This and object-population monotonicity imply that $p\left(R_{K}\right) \subset f_{2}\left(R_{K}, R_{L}, R_{M}\right)$ and $p\left(R_{L}\right) \subset f_{2}\left(R_{K}, R_{L}, R_{M}\right)$. By compatibility, we have $p\left(R_{K}\right) \neq p\left(R_{L}\right)$. Therefore $f_{2}\left(R_{K}, R_{L}, R_{M}\right)=p\left(R_{K}\right) \cup p\left(R_{L}\right)$. But this and object-population monotonicity imply that $f_{1}\left(R_{K}, R_{M}\right)=p\left(R_{K}\right)$, i.e. $R_{K} \succ R_{M}$, the desired conclusion. Therefore $\succ$ is a priority in $\mathbb{P}_{\mathcal{T}}$.

Step 2: Let $\succ$ be a priority defined as in step 1 from $f$. Let $R_{N} \in \mathcal{P}_{1}$. Let $R_{M}$ and $R_{L}$ be distinct maximal peak-unanimous subprofiles of $R_{N}$ such that $R_{M} \succ R_{L}$. Then $f_{1}\left(R_{N}\right) \neq p\left(R_{L}\right)$.

The proof is by induction on $\left|p\left(R_{N}\right)\right|$. For $\left|p\left(R_{N}\right)\right|=2$, the claim follows from the definition of $\succ$. Let $R_{N} \in \mathcal{P}_{1}$ be such that $\left|p\left(R_{N}\right)\right|=n \geq 3$, and suppose that the claim is true for all $R_{N^{\prime}}^{\prime} \in \mathcal{P}_{1}$ such that $\left|p\left(R_{N^{\prime}}^{\prime}\right)\right|=n-1$. Since $n \geq 3$, there is a maximal peak-unanimous subprofile $R_{H}$ of $R_{N}$ distinct from both $R_{L}$ and $R_{M}$, such that $f_{1}\left(R_{N}\right) \neq p\left(R_{H}\right)$. By the induction hypothesis, $f_{1}\left(R_{N \backslash H}\right) \neq p\left(R_{L}\right)$. Let $R_{K}$ be the maximal peak-unanimous subprofile of $R_{N}$, distinct from both $R_{L}$ and $R_{H}$, such that $f_{1}\left(R_{N \backslash H}\right)=p\left(R_{K}\right)$. Strong sovereignty ensures that there exists a peak-unanimous profile $R_{J}$ such that $N \cap J=\emptyset, p\left(R_{J}\right)=p\left(R_{H}\right)$, and $p\left(R_{J}\right) \subset f_{2}\left(R_{N}, R_{J}\right)$. By object-population monotonicity, we have $p\left(R_{K}\right) \subset f_{2}\left(R_{N}, R_{J}\right)$. Since $p\left(R_{K}\right) \neq p\left(R_{J}\right)$, thus $f_{2}\left(R_{N}, R_{J}\right)=p\left(R_{K}\right) \cup p\left(R_{J}\right)$. By object-population monotonicity, we have 
$f_{1}\left(R_{N}\right) \subset p\left(R_{K}\right) \cup p\left(R_{H}\right)$. Therefore, $f_{1}\left(R_{N}\right) \neq p\left(R_{L}\right)$, i.e. the claim is true for $\left|p\left(R_{N}\right)\right|=n$. Thus, it is true for any integer value of $\left|p\left(R_{N}\right)\right|$, which proves the claim.

Step 3: Let $\succ$ be defined as in step 1 from $f$. Let $\left(k, R_{N}\right)$ be a problem. Let $R_{M}$ and $R_{L}$ be distinct maximal peak-unanimous subprofiles of $R_{N}$ such that $R_{M} \succ R_{L}$ and $p\left(R_{L}\right) \subseteq f_{k}\left(R_{N}\right)$. Then $p\left(R_{M}\right) \subseteq f_{k}\left(R_{N}\right)$.

Let $R_{M}$ and $R_{L}$ satisfying the assumptions of the step. Consider all the maximal peak-unanimous subprofiles of $R_{N}$ that are distinct from $R_{M}$ and whose peak location is not contained in $f_{k}\left(R_{N}\right)$. There are at least $m:=\left|p\left(R_{N}\right)\right|-k-1$ such subprofiles. Let $R_{M_{1}}, \ldots, R_{M_{m}}$ be $m$ such subprofiles. By step 2 , we know that $f_{1}\left(R_{M_{1}}, \ldots, R_{M_{m}}, R_{M}, R_{L}\right) \neq p\left(R_{L}\right)$. Therefore $f_{1}\left(R_{M_{1}}, \ldots, R_{M_{m}}, R_{M}, R_{L}\right) \subset$ $p\left(R_{M_{1} \cup \ldots \cup M_{m} \cup M}\right)$. Next, using object-population monotonicity $k-1$ times, we obtain that $f_{k}\left(R_{N}\right) \cap p\left(R_{M_{1} \cup \ldots \cup M_{m} \cup M}\right) \neq \emptyset$. Since $f_{k}\left(R_{N}\right) \cap p\left(R_{M_{1} \cup \ldots \cup M_{m}}\right)=\emptyset$ by definition of the subprofiles $R_{M_{k}}$, therefore $p\left(R_{M}\right) \subseteq f_{k}\left(R_{N}\right)$, the desired conclusion.

It follows from Step 3 that any rule satisfying the axioms is the priority rule associated with $\succ$, where $\succ$ is defined as in Step 1 .

Step 4: Let $\succ$ be the priority associated with a rule $f$. If $f$ is objectpopulation monotonic, then $\succ$ is almost monotonic.

By contradiction, suppose that $R_{M}, R_{K}, R_{H}$ and $R_{L}$ are four peak-unanimous profiles such that $p\left(R_{M}\right)=p\left(R_{L}\right), M \cap L=\emptyset, R_{K}$ and $R_{H}$ are compatible, $R_{M} \succ R_{H} \succ R_{M \cup L}$, and $R_{M} \succ R_{K} \succ R_{M \cup L}$. Then either $R_{H} \succ R_{K}$ or $R_{K} \succ$ $R_{H}$. Suppose, for example, that $R_{H} \succ R_{K}$. Then $f_{1}\left(R_{H}, R_{K}, R_{M}\right)=p\left(R_{M}\right)$ and $f_{2}\left(R_{H}, R_{K}, R_{M \cup L}\right)=p\left(R_{H}\right) \cup p\left(R_{K}\right)$, in violation of object-population monotonicity. The same conclusion is obtained if we assume instead that $R_{K} \succ R_{H}$. Therefore $\succ$ is almost monotonic. 
Step 5: Let $\succ$ be the priority associated with an arbitrary rule $f$. If $f$ is sovereign, then $\succ$ is sovereign.

(i) Let $R_{H}$ and $R_{K}$ be two arbitrary peak-unanimous profiles satisfying $R_{H} \succ R_{K}$, and let $L$ be an arbitrary population. By sovereignty of $f$, since $f_{1}\left(R_{H}, R_{K}\right) \neq p\left(R_{K}\right)$, then there exists a peak-unanimous profile $R_{M}$ satisfying $M \cap(H \cup K \cup L)=\emptyset$, and $f_{1}\left(R_{H}, R_{K}, R_{M}\right)=p\left(R_{M}\right)=p\left(R_{K}\right)$. Since $\left(R_{K}, R_{M}\right)$ is then a peak-unanimous profile, we have $\left(R_{K}, R_{M}\right) \succ R_{H}$. (ii) Next, let $R_{H}$ be an arbitrary peak-unanimous profile, let $x \neq p\left(R_{H}\right)$, and let $L$ be an arbitrary population. By sovereignty of $f$, since $f_{1}\left(R_{H}\right) \neq x$, then there exists a peak-unanimous profile $R_{M}$ satisfying $M \cap(H \cup L)=\emptyset$, and $f_{1}\left(R_{H}, R_{M}\right)=p\left(R_{M}\right)$. Therefore, we have $R_{M} \succ R_{H}$. Therefore $\succ$ is sovereign.

Independence of the axioms We verify that the axioms are independent. First, the left-peaks rule $f^{L P}$ (like any other priority rule whose priority is not sovereign) satisfies all the axioms but sovereignty. Second, the rule $f$ such that $f_{1}$ selects the location $x_{m}$ of the (left)-median voter when $k=1$, and such that $f_{k}$ coincides with the left-peaks rule for all $k \geq 2$ satisfies efficiency and sovereignty, but violates object-population monotonicity. Finally, the rule that locates the first facility on the left-majoritarian peak and the remaining facilities at the smallest $k-1$ positive integers distinct from $p\left(R_{N}\right)$ satisfies object-population monotonicity and sovereignty but violates efficiency. ${ }^{21}$

\footnotetext{
${ }^{21}$ Observe that the three axioms imply object-monotonicity, since all priority rules are object-monotonic. Also, none of the rules characterized in Theorem 1 satisfies populationmonotonicity, since the only population-monotonic priority rules are the left peaks rule and the right peaks rule and these two rules are not sovereign.
} 


\section{Other properties}

In this section and the next one, we investigate on subclasses of priority rules in $\mathcal{F}$ that satisfy other additional desirable properties, such as anonymity, hidingproofness and median-selection. In the next section, we will turn our attention to strategy-proofness.

A rule $f$ satisfies anonymity if, for all $k \geq 1$, and all $R_{N}, R_{M}^{\prime} \in \mathcal{P}_{k}$ where for all $R \in \mathcal{R}$, we have $\left|\left\{i \in M: R_{i}^{\prime}=R\right\}\right|=\left|\left\{i \in N: R_{i}=R\right\}\right|$, we have $f_{k}\left(R_{N}\right)=f_{k}\left(R_{M}^{\prime}\right)$. A priority $\succ$ is anonymous if it satisfies the following condition. For all $R_{M}, R_{N}, R_{M^{\prime}}^{\prime}, R_{N^{\prime}}^{\prime} \in \mathcal{T}$, such that: (i) $R_{M}$ and $R_{N}$ are compatible, (ii) $R_{M^{\prime}}^{\prime}$ and $R_{N^{\prime}}^{\prime}$ are compatible, (iii) for all $R \in \mathcal{R}$, we have $\left|\left\{i \in M: R_{i}=R\right\}\right|=\left|\left\{i \in M^{\prime}: R_{i}^{\prime}=R\right\}\right|$, (iv) for all $R \in \mathcal{R}$, we have $\left|\left\{i \in N: R_{i}=R\right\}\right|=\left|\left\{i \in N^{\prime}: R_{i}^{\prime}=R\right\}\right|$, the following equivalence holds

$$
R_{M} \succ R_{N} \Leftrightarrow R_{M^{\prime}}^{\prime} \succ R_{N^{\prime}}^{\prime}
$$

We leave it to the reader to verify the following result.

Proposition 1: A priority rule $f \in \mathcal{F}$ satisfies anonymity if and only if its priority $\succ$ is anonymous.

Next, we consider a property that ensures that no agent gains from hiding from the social planner. Consider a university which has to choose $k$ software licenses from a larger set of available software for its employees. The decision is taken through an online poll, to which employees can freely respond. One would want that no employee has an incentive not to participate in the poll. A rule $f$ satisfies hiding-proofness if for each problem $k \geq 1$, each $R_{N} \in \mathcal{P}_{k}$, and each $i \in N$ such that $R_{N \backslash\{i\}} \in \mathcal{P}_{k}$, we have $f_{k}\left(R_{N}\right) R_{i} f_{k}\left(R_{N \backslash\{i\}}\right)$. The following holds. 
Proposition 2: A priority rule $f \in \mathcal{F}$ satisfies hiding-proofness if and only if its priority $\succ$ is monotonic.

Proof. The if implication is clear. Let us prove the only if implication. Let $f$ be a priority rule that satisfies hiding-proofness, associated with the priority $\succ$. Let $R_{L}, R_{N} \in \mathcal{T}$ be such that $R_{N}$ and $R_{L}$ are compatible. Let $i \in N$. Suppose that $R_{N \backslash\{i\}} \succ R_{L}$. Then $f_{1}\left(R_{L}, R_{N \backslash\{i\}}\right)=p\left(R_{N \backslash\{i\}}\right)=p\left(R_{N}\right)$. This equality and hiding-proofness imply $f_{1}\left(R_{L}, R_{N}\right)=p\left(R_{N}\right)$, which in turn implies $R_{N} \succ R_{M}$, the desired conclusion.

Last, an important rule for selecting a single location in this model is the one which selects the median peak, for each preference profile $R_{N}$ such that $|N|$ is odd (Black, 1948). A natural question is thus whether there exists a priority rule $f$ such that, for any $R_{N}$ such that $|N|$ is odd, the location $f_{1}\left(R_{N}\right)$ is the median of the peaks. Unfortunately, the answer is negative. In fact we are able to show a much stronger negative result.

Proposition 3: Let $N=\{1, \ldots, 11\}$. There is no rule $f$ satisfying objectpopulation monotonicity and such that, for each $R_{M} \in \mathcal{R}^{M}$, with $M \subset N$ such that $|M|$ is odd, the location $f_{1}\left(R_{M}\right)$ is the median of the peaks of the profile $R_{M}$

Proof: By contradiction, suppose that $f$ is such a rule. Consider a profile $R_{N}$ whose only maximal peak-unanimous subprofiles $R_{\{1,2,3,4\}}, R_{\{5,6\}}, R_{\{8,9,10,11\}}$ have peaks locations respectively at 1,2 and 4 . Let $R_{7}$ be such that $p\left(R_{7}\right)=3$. Then, by the median property, we have $f_{1}\left(R_{N \backslash\{1,2,3,4\}}\right)=4, f_{1}\left(R_{N \backslash\{5,6\}}\right)=3$ and $f_{1}\left(R_{N \backslash\{8,9,10,11\}}\right)=1$. By object-population monotonicity, we have $\{1,3,4\} \subseteq$ $f_{2}\left(R_{N}\right)$, which contradicts $\left|f_{2}\left(R_{N}\right)\right|=2$. 


\section{Strategy-proofness and hierarchical rules}

In this section, we investigate on the class of procedures to locate a single facility that are both the first component of some priority rule and robust to the manipulation of preferences. A 1-rule is a mapping $g: \mathcal{P}_{1} \rightarrow \mathcal{X}_{1}$. It can also be seen as the first component of a rule. A 1-rule is a 1-priority rule if it is the first component of a priority rule. The non-manipulability property we are interested in requires that truthfully reporting preferences be a (weakly) dominant strategy in the associated direct revelation game:

A 1-rule $g$ satisfies strategy-proofness if for each $R_{N} \in \mathcal{P}_{1}$, each $i \in N$ and each $R_{i}^{\prime} \in \mathcal{R}$, we have $g\left(R_{N}\right) R_{i} g\left(R_{i}^{\prime}, R_{-i}\right)$.

The first components of the left-peaks rule, the right-peaks rule and of all the serial dictatorships are strategy-proof priority 1-rules. More generally, the first component of any hierarchical rule (see Example 8) is strategy-proof. In fact, we will show in this section that the hierarchical 1-rules are the only strategy-proof priority 1-rules. ${ }^{22}$

\subsection{Strategy-proof priority $N$-schemes}

For each population $N$, an $N$-scheme is a mapping $g_{N}: \mathcal{R}^{N} \rightarrow \mathcal{X}_{1}$. It can also be seen as the restriction of a 1-rule to the subset $\mathcal{R}^{N}$. Moulin (1980) introduced the following class of $N$-schemes.

An $N$-scheme $g_{N}$ is a peak-selecting generalized median $N$-scheme if there is a list $\left\{a_{M}\right\}_{M \subseteq N}$ of parameters such that $a_{N}=-\infty, a_{\emptyset}=+\infty$, and for each $M \subseteq N$, we have $a_{M} \in\{-\infty,+\infty\}$; for all $L, M \subseteq N$, we have $L \subseteq M \Rightarrow a_{M} \leq$

\footnotetext{
${ }^{22}$ The definition of strategy-proofness can be extended to rules in the obvious way. See the comments at the end of the section.
} 
$a_{L}$; and for each $R_{N} \in \mathcal{R}^{N}$, we have

$$
g\left(R_{N}\right)=\min _{M \subseteq N}\left\{\max _{i \in M}\left[a_{M}, p\left(R_{i}\right)\right]\right\} .
$$

It is well known that peak-selecting generalized median $N$-schemes are the only peak selecting strategy-proof $N$-schemes. ${ }^{23}$ Let $\mathcal{M}_{N}$ be the class of peakselecting generalized median $N$-schemes. We say that a subset $M \subseteq N$ is minimal for the $N$-scheme $g$ if $a_{M, N}=-\infty$ and, for all $L \varsubsetneqq M$, we have $a_{L}=+\infty$. In particular, any minimal subset $M$ is nonempty, and is therefore a subpopulation of $N$. Clearly, we have

$$
g\left(R_{N}\right)=\min _{\substack{M \subseteq N \\ \text { s.t. } M \text { is minimal }}}\left\{\max \left[p\left(R_{M}\right)\right]\right\} .
$$

We say that an $N$-scheme $g_{N}$ is a priority $N$-scheme if there is a priority 1-rule that coincides with $g_{N}$ on $\mathcal{R}^{N}$.We now provide a description of all strategy-proof priority $N$-schemes, in terms of the collection of their minimal sets.

Lemma 3: Let $g_{N}$ be a strategy-proof priority $N$-scheme. Then, $g_{N}$ is peakselecting generalized median. Furthermore, either $g_{N}$ has exactly one minimal set, or all minimal sets of $g_{N}$ are singletons.

Proof: Since $g_{N}$ is a priority $N$-scheme, it satisfies peaks-selection. Since it is also strategy-proof, then it is a peak-selecting generalized median. Therefore, it can be described by its minimal sets. By contradiction, suppose that $L$ and $M$ are two distinct minimal sets for $g_{N}$, and that $|M| \geq 2$. Let $j^{*} \in M \backslash L$. Since $|M| \geq 2$, then $M \backslash\left\{j^{*}\right\} \neq \emptyset$. Since $L$ and $M$ are two distinct minimal sets for $g_{N}$, then $L \nsubseteq M$, which further implies that $N \backslash M \neq \emptyset$. The sets $\left\{j^{*}\right\}$, $M \backslash\left\{j^{*}\right\}$, and $N \backslash M$ form a disjoint partition of $N$, and none of these sets is empty.

\footnotetext{
${ }^{23}$ This follows from the work of Moulin (1980) and Barberà and Jackson (1994). This family plays an important role in the work of Jackson and Nicolò (2004), and Cantala (2004).
} 
Let $R_{N} \in \mathcal{R}^{N}$ be a profile whose maximal peak-unanimous subprofiles are $R_{M \backslash\left\{j^{*}\right\}}, R_{N \backslash M}$ and $R_{j^{*}}$ and have peak locations respectively at 0,1 and 2, so that $p\left(R_{N}\right)=\{0,1,2\}$. Since $M$ is minimal, then for each minimal set $K$, we do not have $K \varsubsetneqq M$. In particular, we do not have $K \subseteq M \backslash\left\{j^{*}\right\}$. Therefore $\max \left[p\left(R_{K}\right)\right] \geq 1$. Since this holds for each minimal set $K$, therefore $g_{N}\left(R_{N}\right) \geq 1$. Since, however, $\max \left[p\left(R_{L}\right)\right]=1$ and $L \subseteq N \backslash\{j\}$, then $g_{N}\left(R_{N}\right)=1$. Thus, $R_{N \backslash M} \succ R_{M \backslash\left\{j^{*}\right\}}$.

Next, let $R_{j^{*}}^{\prime}$ be a preference whose peak location is at -1 . Let $R_{N}^{\prime}:=$ $\left(R_{j^{*}}^{\prime}, R_{N \backslash\left\{j^{*}\right\}}\right)$. Then, we have $p\left(R_{N}^{\prime}\right)=\{-1,0,1\}$. Since $M$ is minimal, then for each minimal set $K$, we do not have $K \varsubsetneqq M$. In particular, we do not have $K \subseteq\left\{j^{*}\right\}$. Therefore $\max \left[p\left(R_{K}^{\prime}\right)\right] \geq 0$. Since this holds for each minimal set $K$, therefore $g_{N}\left(R_{N}^{\prime}\right) \geq 0$. Since, however, $\max \left[p\left(R_{M}^{\prime}\right)\right]=0$, then $g_{N}\left(R_{N}^{\prime}\right)=0$. Therefore, $R_{M \backslash\left\{j^{*}\right\}}^{\prime} \succ R_{N \backslash M}^{\prime}$, i.e. $R_{M \backslash\left\{j^{*}\right\}} \succ R_{N \backslash M}$, which contradicts $R_{N \backslash M} \succ$ $R_{M \backslash\left\{j^{*}\right\}} \cdot$

We now provide a convenient description of the family of strategy-proof priority $N$-schemes. Consider a pair $(C, D)$ such that $C \subseteq N$ and $D \subseteq N$. Let the $N$-scheme $g_{N}$ be the $(C, D)$-oligarchy if exactly one of the following mutually exclusive conditions holds.

(a). $|C| \geq 2, D=\emptyset$, and for all $R^{N} \in \mathcal{R}^{N}, \quad g_{N}\left(R_{N}\right)=\min \left\{p\left(R_{C}\right)\right\}$.

(b). $C=\emptyset,|D| \geq 2$, and for all $R^{N} \in \mathcal{R}^{N}, \quad g_{N}\left(R_{N}\right)=\max \left\{p\left(R_{D}\right)\right\}$.

(c). $C=D=\left\{i^{*}\right\}$ for some $i^{*} \in N$, and for all $R^{N} \in \mathcal{R}^{N}, \quad g_{N}\left(R_{N}\right)=p\left(R_{i}\right)$.

The $(C, D)$-oligarchy is not defined for all pairs $(C, D)$, only the ones satisfying one of the above admissibility conditions. Also, two distinct pairs $(C, D)$ and $\left(C^{\prime}, D^{\prime}\right)$ define different oligarchies. In case $(a)$, the scheme is similar to a left-peaks $N$-scheme, but it only takes into account a subset $C$ of the population. In case (b), it is similar to a right-peaks $N$-scheme, but it takes into 
account only a subset $D$ of the population. In case (c), it is the dictatorship of some agent $i^{*}$. As we will see in Theorem 2, an $N$-scheme is a strategy-proof priority $N$-scheme if and only if it is a $(C, D)$-oligarchy for some admissible pair $(C, D)$.

\subsection{Conjugate weak partial orders}

In order to be able to characterize strategy-proof priority 1-rules, we introduce here a few concepts. Two weak partial orders $\unrhd_{L}$ and $\unrhd_{R}$ on $\mathbb{N}$ are conjugate if for all $i, j \in \mathbb{N}$ such that $i \neq j$, we have $\left(i \unrhd_{L} j\right) \Leftrightarrow \neg\left(j \unrhd_{R} i\right)$. Given two weak partial orders $\unrhd^{\prime}$ and $\unrhd^{\prime \prime}$ on $\mathbb{N}$, let the union of $\unrhd^{\prime}$ and $\unrhd^{\prime \prime}$ be the binary relation $\unrhd$ on $\mathbb{N}$ such that, for all $i, j \in \mathbb{N}$, we have $i \unrhd j \equiv\left(i \unrhd^{\prime} j\right.$ or $\left.i \unrhd^{\prime \prime} j\right)$. The following holds.

Lemma 4: Let $\unrhd_{L}$ and $\unrhd_{R}$ be two conjugate partial orders on $\mathbb{N}$. Then the union $\unrhd_{\text {of }} \unrhd_{L}$ and $\unrhd_{R}$ is a weak ordering.

Proof. We leave it to the reader to verify that the relation $\unrhd$ is reflexive and complete. We will prove that it is transitive. Let $i, j, k$ be arbitrary elements of $\mathbb{N}$, such that $i \unrhd j$, and $j \unrhd k$. We will prove that $i \unrhd k$. If $i, j$ and $k$ are not three distinct integers, the claim is obviously true. Suppose then that $i, j$ and $k$ are three distinct integers. Four cases may arise. Case 1: $i \unrhd_{L} j$, and $j \unrhd_{L} k$. Then by transitivity of $\unrhd_{L}$, we have $i \unrhd_{L} k$. Case 2: $i \unrhd_{R} j$, and $j \unrhd_{R} k$. Then by transitivity of $\unrhd_{R}$, we have $i \unrhd_{R} k$. Case 3: $i \unrhd_{L} j$, and $j \unrhd_{R} k$. Since $i \unrhd_{L} j$, $i \neq j$, and $\unrhd_{L}$ and $\unrhd_{R}$ are conjugates, therefore $\neg\left(j \unrhd_{R} i\right)$. By transitivity of $\unrhd_{R}$, and since $j \unrhd_{R} k$ and $\neg\left(j \unrhd_{R} i\right)$, therefore $\neg\left(k \unrhd_{R} i\right)$. Since $k \neq i$, and $\unrhd_{L}$ and $\unrhd_{R}$ are conjugates, this is equivalent to $i \unrhd_{L} k$. Case $4: i \unrhd_{R} j$, and $j \unrhd_{L} k$. Following a reasoning symmetric to Case 3 , we can prove that $i \unrhd_{R} k$. In all four cases we proved, either that $i \unrhd_{L} k$, or that $i \unrhd_{R} k$. Therefore, in all four cases, $i \unrhd k$, the desired conclusion, holds. 
Lemma 5: Let $\unrhd_{L}$ and $\unrhd_{R}$ be two conjugate weak partial orders. Let $T \subset \mathbb{N}$ be an indifference class for the union $\unrhd_{\text {of }} \unrhd_{L}$ and $\unrhd_{R}$ with at least two elements. Then, $T$ is an indifference class, either for $\unrhd_{L}$, or for $\unrhd_{R}$, but not for both.

Proof. Throughout the proof, let $\bowtie, \bowtie_{L}$, and $\bowtie_{R}$ denote the respective indifference relations associated with $\unrhd_{,} \unrhd_{L}$ and $\unrhd_{R}$. We will first show that for all $i, j \in T$, such that $i \neq j$, and $i \bowtie j$, we have either $i \bowtie_{L} j$ or $i \bowtie_{R} j$, but not both. Let $i, j \in T$ be two such agents. Since $\unrhd_{L}$ and $\unrhd_{R}$ are conjugates and $i \neq j$, we cannot have $i \unrhd_{L} j$ and $j \unrhd_{R} i$. For the same reason, we cannot have $i \unrhd_{R} j$ and $j \unrhd_{L} i$. Therefore, either $i \bowtie_{L} j$, or $i \bowtie_{R} j$, but not both. If $T$ has exactly two elements, there is nothing left to prove. Assume then that $T$ has at least three elements.

Next, we will show that for all three distinct $i, j, k \in T$, we have either $i \bowtie_{L} j \bowtie_{L} k$, or $i \bowtie_{R} j \bowtie_{R} k$, but not both. We will first show that $i \bowtie_{L} j \bowtie_{R} k$ cannot hold. Suppose, by contradiction, that these relations hold. Then, on the one hand, we have $i \unrhd_{L} j \unrhd_{R} k$. By Case 3 in the proof of Lemma 4, this implies that $i \unrhd_{L} k$. But, also, on the other hand, we have $k \unrhd_{R} j \unrhd_{L} i$. By Case 4 in the proof of Lemma 4 , this implies that $k \unrhd_{R} i$. Thus we have $i \neq k$, $i \unrhd_{L} k$ and $k \unrhd_{R} i$, which contradicts that $\unrhd_{L}$ and $\unrhd_{R}$ are conjugates. Therefore, $i \bowtie_{L} j \bowtie_{R} k$ cannot hold. A symmetric reasoning shows that $i \bowtie_{R} j \bowtie_{L} k$ cannot hold either. Therefore, we have either $i \bowtie_{L} j \bowtie_{L} k$, or $i \bowtie_{R} j \bowtie_{R} k$, but not both. Since this holds for all distinct $i, j, k \in T$, it follows that $T$ is an indifference class, either for $\unrhd_{L}$, or for $\unrhd_{R}$, but not for both, the desired conclusion.

\subsection{Strategy-proof priority 1-rules}

We are now ready to provide a characterization of all strategy-proof priority 1-rules. We describe this set in two ways.

Under the first description, each such 1-rule is viewed as the collection of 
the $N$-schemes it induces: Each of them is a $\left(C_{N}, D_{N}\right)$-oligarchy. Moreover the parameters $\left(C_{N}, D_{N}\right)$ are linked across population, in a way which will be made clear. We describe the exact nature of the linkage. In the second description, we focus on the priority itself and show that it is hierarchical, as defined in Example 4. We are now ready to present the main result of this section.

Theorem 2: Let $g$ be a 1-rule. The three following statements are equivalent.

(i). $g$ is a strategy-proof priority 1-rule.

(ii). There are conjugate weak partial orders $\unrhd_{L}$ and $\unrhd_{R}$ such that, for each population $N$, the 1-rule $g$ induces the $\left(C_{N}, D_{N}\right)$-oligarchic $N$-scheme $g_{N}$ on $N$, with $C_{N}=\left\{i \in N: \forall j \in N, i \unrhd_{L} j\right\}$ and $D_{N}=\{i \in N: \forall j \in$ $\left.N, i \unrhd_{R} j\right\}$.

(iii). $g$ is a hierarchical 1-rule.

Proof: $($ iii $) \Longrightarrow(i)$. A hierarchical rule $g$ is obviously a priority 1-rule. It remains to show that it is strategy-proof. Let $N$ be an arbitrary population. Let $g_{N}$ be the $N$-scheme induced by $g$ on $N$. Let $T$ be the highest priority class of agents in the hierarchy such that $T \cap N \neq \emptyset$. Then either, for all profile $R_{N} \in \mathcal{R}^{N}$, we have $g_{N}\left(R_{N}\right)=\min \left[p\left(R_{N \cap T}\right)\right]$ or, for all profile $R_{N} \in \mathcal{R}^{N}$, we have $g_{N}\left(R_{N}\right)=\max \left[p\left(R_{N \cap T}\right)\right]$. In both cases, the $N$-scheme $g_{N}$ is strategyproof, the desired conclusion.

$(i) \Longrightarrow(i i)$. By Lemma 3, either $g_{N}$ has a single minimal set and this set is not a singleton, or it contains more than one minimal set and they are all singletons, or it contains a single set and it is a singleton. Clearly, these three cases are mutually exclusive. In the first case, let $C_{N}$ be the union of the many singleton sets for $g_{N}$, and let $D_{N} \equiv \emptyset$. In the second case, let $D_{N}$ be unique (non singleton) minimal set, and let $C_{N} \equiv \emptyset$. In the third and last case, let $i^{*}$ be the 
unique element of the unique minimal set, and let $C_{N} \equiv\left\{i^{*}\right\}$, and $D_{N} \equiv\left\{i^{*}\right\}$. Clearly, for each $N$, the pair $\left(C_{N}, D_{N}\right)$ satisfies conditions $(a),(b)$ and $(c)$ of the definition of an oligarchic 1-rule. Next, let $\unrhd_{L}$ and $\unrhd_{R}$ be two binary relations on $\mathbb{N}$ defined as follows. For all $i, j \in \mathbb{N}$, let $\left(i \unrhd_{L} j\right) \Leftrightarrow\left(i \in C_{\{i, j\}}\right.$ or $\left.i=j\right)$, and let $\left(i \unrhd_{R} j\right) \Leftrightarrow\left(i \in D_{\{i, j\}}\right.$ or $\left.i=j\right)$. We will prove that $\unrhd_{L}, \unrhd_{R}$ satisfy the requirements of statement (ii) in three steps.

Step 1. The binary relations $\unrhd_{L}$ and $\unrhd_{R}$ are transitive. We will only prove that $\unrhd_{L}$ is transitive. The proof that $\unrhd_{R}$ is transitive follows a symmetric reasoning. Let $i, j$ and $k$ be three agents such that $i \unrhd_{L} j$ and $j \unrhd_{L} k$. We claim that $i \unrhd_{L} k$ holds. If these agents are not all distinct, then our claim is obvious. Suppose then that they are distinct. Let the preferences $R_{i}, R_{j}$ and $R_{k}$ have their respective peak locations at $-1,0$ and 1 . Then, on the one hand, $i \unrhd_{L} j$ and $i \neq j$ imply that $i \in C_{\{i, j\}}$. Since $p\left(R_{i}\right)=-1<0=p\left(R_{j}\right)$, it follows that $g\left(R_{i}, R_{j}\right)=-1$. Since $g$ is the priority rule associated with $\succ$, this implies that $R_{i} \succ R_{j}$. By a similar reasoning, we can show that $j \unrhd_{L} k$ implies that $R_{j} \succ R_{k}$. By almost transitivity, we obtain $R_{i} \succ R_{k}$. Therefore, $g\left(R_{i}, R_{k}\right)=-1$. Since $p\left(R_{i}\right)=-1<1=p\left(R_{k}\right)$, this implies that $i \in C_{\{i, k\}}$. Therefore, we have $i \unrhd_{L} k$. Therefore, $\unrhd_{L}$ is transitive, the desired conclusion.

Step 2. The weak partial orders $\unrhd_{L}$ and $\unrhd_{R}$ are conjugate. Let $i, j \in N$ such that $i \neq j$ and $i \unrhd_{L} j$. Then $i \in C_{\{i, j\}}$ and $i \neq j$. Therefore $D_{\{i, j\}} \subseteq\{i\}$. In particular, $j \notin D_{\{i, j\}}$ and $i \neq j$. Therefore, $\neg\left(j \unrhd_{R} i\right)$, the desired conclusion.

Step 3. For all $N$, we have $C_{N}=\left\{i \in N: \forall j \in N, i \unrhd_{L} j\right\}$ and $D_{N}=$ $\left\{i \in N: \forall j \in N, i \unrhd_{R} j\right\}$. We will only prove the claim for $C_{N}$. The proof of the claim for $D_{N}$ follows a symmetric reasoning. First we prove that $C_{N} \subseteq$ $\left\{i \in N: \forall j \in N, i \unrhd_{L} j\right\}$. Let $i \in C_{N}$ and $j \in N$. We will prove that $i \unrhd_{L} j$. Let $R_{N}$ be a profile whose only maximal peak-unanimous subprofiles are $R_{i}$, $R_{j}$ and $R_{N \backslash\{i, j\}}$ and have their respective peak locations at $-1,0$ and 1 . Then $g\left(R_{N}\right)=\min \left[p\left(R_{C_{N}}\right)\right]=-1$. This implies that $R_{i} \succ R_{j}$. Therefore, $g\left(R_{i, j}\right)=$ 
-1, i.e. $i \unrhd_{L} j$. Second, we prove that $\left\{i \in N: \forall j \in N, i \unrhd_{L} j\right\} \subseteq C_{N}$. Let $i \in\left\{i \in N: \forall j \in N, i \unrhd_{L} j\right\}$. We will prove that $i \in C_{N}$. Let $j_{1}, \ldots, j_{l}$ be the elements of $N \backslash i$. Let $R_{N}$ be a profile such that $p\left(R_{i}\right):=0$ and for all $k=1, \ldots, l$, we have $p\left(R_{j_{l}}\right):=l$. For all $k=1, \ldots, l$, we have $i \unrhd_{L} j_{k}$, therefore $g\left(R_{i, j_{k}}\right)=-1$, therefore $R_{i} \succ R_{j_{k}}$. Since this holds for all $k=1, \ldots, l$ and $g$ is a priority rule, it follows that $g\left(R_{N}\right)=-1=p\left(R_{i}\right)$. Therefore $i \in C_{N}$, the desired conclusion.

(ii) $\Longrightarrow($ iii). Let the statement (ii) hold. We will proceed in two steps. First, we will construct a candidate hierarchical priority $\succ$. Second, we will show that $g$ is indeed the 1-rule associated with this priority.

Step 1: Definition of a candidate hierarchical priority. Let $\succ$ be binary relation on $\mathcal{T}$ defined as follows. Let $\unrhd$ be the union of $\unrhd_{L}$ and $\unrhd_{R}$. Then $\unrhd$ is a weak ordering of $\mathbb{N}$, such that each indifference class $W$ for $\unrhd$ is either an indifference class for $\unrhd_{L}$, or for $\unrhd_{R}$, but not for both. Let $\succeq$ be the weak ordering on $\mathcal{T}$ defined as follows. For all $R_{M}, R_{N} \in \mathcal{T}$, we have $R_{M} \succeq R_{N}$ if there is $i \in M$ such that for all $j \in N \cup M$, we have $i \unrhd j$. Let $\mathbb{T}^{*}$ be the set of non-singleton indifference classes for $\succeq$ in $\mathcal{T}$. Then, for each non singleton indifference class $T \in \mathbb{T}^{*}$, there is a (unique) indifference class $W_{T}$ for $\unrhd$ in $\mathbb{N}$, such that $T=\left\{R_{N} \in \mathcal{T}: N \cap W_{T} \neq \emptyset\right\}$. We know that for each class $T \in \mathbb{T}^{*}$, the class $W_{T}$ satisfies exactly one of the following two mutually exclusives conditions. Either it is a non-singleton indifference class for $\unrhd_{L}$, or it is a non-singleton indifference class for $\unrhd_{R}$. We will now define a priority $\succ_{T}$ over each non-singleton indifference class $T \in \mathbb{T}^{*}$. If $W_{T}$ is a non-singleton indifference class for $\unrhd_{L}$, then let $\succ_{T}$ be the left peaks priority over $T$. If instead $W_{T}$ is a non-singleton indifference class for $\unrhd_{L}$, then let $\succ_{T}$ be the right peaks priority over $T$. The tuple $\left(\succeq,\left(\succ_{T}\right)_{T \in \mathbb{T}}\right)$ defines a hierarchical priority $\succ$.

Step 2: $g$ is the priority 1-rule associated with $\succ$. We know that each of the $N$-schemes $g_{N}$ satisfies peaks selection. Thus $g$ satisfies peaks selection. Let 
$R_{N}$ be an arbitrary profile. Let $R_{L}$ be the maximal peak-unanimous subprofile of $R_{N}$ such that $g\left(R_{N}\right)=p\left(R_{L}\right)$. We will show that this implies that for all other maximal peak-unanimous subprofile $R_{M}$ of $R_{N}$, we have $R_{L} \succ R_{M}$. To do so, we will distinguish three mutually exclusive cases. Case 1: $\left|C_{N}\right| \geq 2$. Then $p\left(R_{L}\right)=\min \left\{p\left(R_{C_{N}}\right)\right\}$. Since it is nonempty, the set $C_{N}$ is the highest indifference class for $\unrhd$ in $N$ and is also an indifference class for $\unrhd_{L}$. This and $p\left(R_{L}\right)=\min \left\{p\left(R_{C_{N}}\right)\right\}$ implies that $R_{L} \succ R_{M}$, for all maximal peakunanimous profiles $R_{M}$ in $R_{N}$ that are distinct from $R_{L}$. Case 2: $\left|D_{N}\right| \geq 2$. Then $p\left(R_{L}\right)=\max \left\{p\left(R_{D_{N}}\right)\right\}$. Since it is nonempty, the set $D_{N}$ is the highest indifference class for $\unrhd$ in $N$ and is also an indifference class for $\unrhd_{R}$. This and $p\left(R_{L}\right)=\min \left\{p\left(R_{C_{N}}\right)\right\}$ implies that $R_{L} \succ R_{M}$, for all maximal peak-unanimous profiles $R_{M}$ in $R_{N}$ that are distinct from $R_{L}$. Case 3: $C_{N}=D_{N}=\{i\}$ for some $i \in N$. Then $i$ is the sole element in the highest indifference class for $\unrhd$ in $N$. As a consequence, whichever maximal peak-unanimous profile of $R_{N}$ has agent $i$ enjoys the highest priority for $\succ$. Since $g\left(R_{N}\right)=p\left(R_{L}\right)$, therefore $i \in L$. Therefore, $R_{L} \succ R_{M}$, for all maximal peak-unanimous subprofiles $R_{M}$ of $R_{N}$ that are distinct from $R_{L}$. Therefore, $g$ is the hierarchical 1-rule associated with the priority $\succ$, the desired conclusion.

Finally, we should point out that, although we defined strategy-proofness for 1-rules, the definition can easily be adapted for rules. It is easy to see that hierarchical rules are strategy-proof. Thus, our results in this section also imply that the only strategy-proof priority rules are the hierarchical rules.

We conclude with the following additional observations. All of the hierarchical rules satisfy hiding-proofness. However, the left-peaks rule and the right-peaks rule are the only anonymous hierarchical rules. Each majoritarian rule is hiding-proof and anonymous, but it is not a hierarchical rule, i.e. it is not strategy-proof. All hierarchical priorities are monotone, therefore almost monotone. A necessary and sufficient condition for a hierarchical priority $\succ$ to 
be sovereign is that the weak ordering $\unrhd$ of the agents that is associated with it should have no maximal element.

\section{Conclusion}

In this paper we undertake the task of designing rules for the provision of multiple public facilities to populations of agents with single peaked preferences over the real line. To this end, we introduce two new axioms-object-population monotonicity and sovereignty. We uncover and characterize a rich family of priority rules. Some rules in the family are appealing, for example the majoritarian rules. Unfortunately, no priority rule coincides with the median for each problem of providing exactly one public facility to an odd population of agents. On the positive side, the structure of the rules in the family is very simple, which makes it possible to characterize several subfamilies on the basis of anonymity, hiding-proofness and strategy-proofness. In particular, strategy-proof priority rules form the interesting subfamily of hierarchical rules.

We view our work as an initial step towards a comprehensive understanding of the implications of normative and strategic axioms in the problem of locating multiple facilities. An obvious question is whether it is possible to generalize Moulin's (1980) generalized median voter schemes to this more complex setting. We only provide a partial answer to this question. The priority structure enables us to generalize only a particular type of generalized median voter scheme, the oligarchies. A generalization of the entire family of generalized median voter schemes to the problem of locating multiple facilities requires a flexibility which conflicts with the priority structure, and thus with our main axioms.

Our analysis is based on a particular way to extend preferences over singlelocations to preferences over multiple locations, the max-extension, introduced by Miyagawa $(1998,2001)$. The work of Miyagawa seems to indicate that the max-extension is not very tractable, in the sense that it leads to characteriza- 
tions of rules that are not particularly appealing, such as the left peaks rule and the right peaks rule, and other more complicated rules. As our results suggest, this may have more to do with the solidarity axioms used there than the max-extension itself. As Gordon (2007a) has shown, solidarity axioms have strong general implication in all public decisions models, of which the location of multiple public facilities is a special case. Nevertheless, both Miyagawa and us obtain highly discontinuous rules, for any natural topology. This seems to be a regular feature of the max-extension model. Thus, insisting on continuity in this model may be too strong a requirement. Majoritarian rules, for instance, are not continuous.

However, a natural extension of our work would be precisely to study what happens under other types of preference extensions, e.g. the lexicographic extension. Ehlers $(2002,2003)$ studies the same axioms as Miyagawa $(1998,2001)$ under the lexicographic extension and obtains strikingly different characterizations. So, it is quite possible that the axioms we used here would lead to other families of rules as well, if one were to replace the max-extension of our model with the lexicographic extension, or some other extension.

Finally, we have considered here the pure public goods case. Richer set-ups include problems with congestion and cost-sharing to finance the facilities, such as in the model by Jackson and Nicolò (2004), for the case $k=1$. We leave this and the other questions open for future research.

\section{References:}

1. Barberà S., Beviá C., 2002. "Self-Selection Consistent Functions." Journal of Economic Theory, 105, 263-277.

2. Barberà S., Beviá C., 2005. "Locating Public Facilities by Majority: Stability, Consistency and Group formation." Games and Economic Behavior, 56, 185-200.

3. Barberà S., Jackson M., 1994. "A Characterization of Strategy-Proof 
Social Choice Functions for Economies with Pure Public Goods." Social Choice and Welfare, 11, 241-252.

4. Black D., 1948. "On the Rationale of Group Decision-Making." Journal of Political Economy, 56, 23-34.

5. Bochet O., Gordon S., 2008, "Priorities in the Location of Multiple Public Facilities," CIREQ working paper, Université de Montréal.

https://www.webdepot.umontreal.ca/Usagers/gordons/MonDepotPublic/wp.pdf

6. Cantala D. 2004. "Choosing the Level of a Public Good when Agents have an Outside Option." Social Choice and Welfare, 22, 491-514.

7. Ching S., 1997. "Strategy-Proofness and Median Voters." International Journal of Game Theory, 26, 473-490.

8. Ching S. and Thomson W., 1996. "Population-Monotonic Solutions in Public Good Economies with Single-Peaked Preferences." Forthcoming, Social Choice and Welfare.

9. Ehlers L., 2002. "Multiple Public Goods and Lexicographic Preferences." Journal of Mathematical Economics, 37, 1-15.

10. Ehlers L., 2003. "Multiple Public Goods, Lexicographic Preferences, and Single-Plateaued Preference Rules." Games and Economic Behavior, 43, $1-27$.

11. Fidrmuc, J., Ginsburgh V., Weber S., 2006. "On the Choice of Official Languages in the European Union." mimeo.

12. Gordon S., 2007a. "Public Decisions: Solidarity and the Status Quo." Games and Economic Behavior, 61, 225-241.

13. Gordon S., 2007b. "Solidarity in Choosing a Location on a Cycle." Social Choice and Welfare, 29, 125-147.

14. Jackson M., Nicolò A., 2004. "The Strategy-Proofness provision of Public Goods under Congestion and Crowding Preferences." Journal of Economic Theory, 115, 278-308. 
15. Ju B., 2008. "Efficiency and Consistency for Locating Multiple Public Facilities." Journal of Economic Theory, 138, 165-183.

16. Klaus B., 2001. "Target Rules for Public Choice Economies on Tree Networks and in Euclidean Spaces." Theory and Decision, 51, 13-29.

17. Miyagawa E., 1998. "Mechanisms for Providing a Menu of Public Goods." PhD dissertation, University of Rochester.

18. Miyagawa E., 2001. "Locating Libraries on a Street." Social Choice and Welfare, 18, 527-541.

19. Moulin H., 1980. "On Strategy-Proofness and Single-Peakedness." Public Choice, 35, 437-455.

20. Thomson, W., 1993. "The Replacement Principle in Public Good Economies with Single-Peaked Preferences." Economic Letters, 42, 31-36.

21. Thomson, W., 1995. "Population-Monotonic Allocation Rules." In: Barrett W., Moulin H., Salles M., Schofield N. (eds.), Social Choice, Welfare, and Ethics, Cambridge University Press, Cambridge, pp. 79-124.

22 Thomson, W., 1999a. "Welfare-domination under Preference-Replacement: a Survey and Open Questions." Social Choice and Welfare, 16, 373-394.

23 Thomson, W., 1999b. "Population-Monotonicity: a Survey and Open Questions." Working Paper, University of Rochester.

24. Vohra, R. V., 1999. "The Replacement principle and Tree Structured Preferences." Economic Letters, 63, 175-180. 